\title{
Research on IQ Imbalance Error of Orthogonal Alternating Sampling
}

\author{
Junyi Luo $\mathbb{D D}^{1}$ and Tao Liu $\mathbb{D}^{2}$ \\ ${ }^{1}$ School of Electronic Information and Electrical Engineering, Chengdu University, Chengdu 610106, China \\ ${ }^{2}$ Chengdu Kinyea Technologies Co., Ltd, Chengdu 610106, China
}

Correspondence should be addressed to Tao Liu; 179209457@qq.com

Received 13 January 2022; Accepted 11 February 2022; Published 4 March 2022

Academic Editor: Zi-Peng Wang

Copyright ( $) 2022$ Junyi Luo and Tao Liu. This is an open access article distributed under the Creative Commons Attribution License, which permits unrestricted use, distribution, and reproduction in any medium, provided the original work is properly cited.

\begin{abstract}
In the signal acquisition of complete orthogonal frequency alternating acquisition system, we need to transform the broadband input signal to baseband through analogue orthogonal demodulation. Thus, the IQ imbalance errors will inevitably exist, including amplitude imbalance error and phase imbalance error, resulting in distortion deterioration of reconstruction signals. This paper makes an in-depth study on such IQ imbalance errors and mathematically deduces the mathematical expressions of imbalance errors and the spurious frequency distribution caused by them, which shows that the positions where the spurious signals appear are closely related to the frequency of input signal and the tuning frequency of analogue quadrature demodulation. Meanwhile, the amplitude and phase imbalance parameters of the channels I and Q are also calculated. Finally, the spurious effects caused by imbalance errors are verified by simulation experiment, and the verification results are consistent with the theoretical derivation results.
\end{abstract}

\section{Introduction}

Sampling rate and bandwidth are two key indicators to evaluate the performance of a set of data acquisition systems, and the two indicators are also directly reflected in the performance of analogue-to-digital converter (ADC). At the current level of integrated circuit technology, the sampling rate and bandwidth of a single ADC is extremely limited; in order to build a wideband high-speed acquisition system, it can only be realized by combination sampling using several ADCs. One combination mode is time-interleaved sampling, namely, TIADC mode $[1,2]$, which can effectively break the limit of sampling rate, but the bandwidth is still limited; the other combination mode is frequency-interleaved sampling, namely, FIADC mode [3], also known as the HBF mode [4], which is the best means to effectively increase the sampling rate and bandwidth.

The structural forms of frequency-interleaved sampling technology mainly include two types, namely, traditional structure [5] and orthogonal structure [6, 7]. The orthogonal structure is an equivalent improvement of the traditional structure. Its concept is that the input signal is uniformly divided into several sub-bands in terms of frequency spectrum characteristics, each sub-band is moved to the baseband by analogue IQ quadrature demodulation, and then the various baseband signals are sampled using the $\mathrm{ADC}$ and the carrier frequency of these baseband signals is recovered with the help of the digital IQ quadrature modulation algorithm, so as to achieve the purpose of reproducing the characteristics of input signal. As this structure involves the two characteristic links of analogue IQ quadrature demodulation and digital IQ quadrature modulation, it is also known as a complete orthogonal frequency alternating acquisition structure. In this structure, it required to design only one analogue low-pass filter at the front end of the ADC array and one digital low-pass filter at the back end, which greatly reduces the design complexity and error sources of filters and is more conducive to engineering applications; moreover, the sampling rate and bandwidth of various $\mathrm{ADCs}$ are further reduced by a half compared to 
those of the traditional structure, which is conducive to construction of higher speed and larger bandwidth acquisition systems.

The errors existing in both traditional and complete orthogonal frequency alternating acquisition systems include analogue device error, channel mismatch error, and signal reconstruction error. At present, a large number of literatures [8-11] have made an in-depth study on evaluation, compensation, calibration, etc. of these errors. However, due to the introduction of an analogue IQ quadrature demodulator at the front end of the ADC, the IQ imbalance errors will inevitably exist in the complete orthogonal frequency alternating structure $[12,13]$, including the two parts of IQ amplitude imbalance error and phase imbalance error, which directly leads to frequency spectrum deterioration and spurious cluttering of output signals. The study on IQ imbalance errors in such structures is still quite scarce and needs to be further developed. This paper focuses on studying the IQ imbalance errors in the complete orthogonal frequency alternating systems, progressively deduces the mathematical expression of the output spurious signals in the case of IQ imbalance, determines the frequency positions of various spurious signals and their correlations with input signal frequency, obtains the computation expressions of amplitude and phase imbalance parameters, and finally verifies the analysis by simulation experiment.

\section{Principle of Complete Orthogonal Frequency Alternating Acquisition}

Figure 1 shows the principle structure of complete orthogonal frequency alternating acquisition system, of which sampling period is $T_{s}$.

The analogue quadrature demodulator and analogue low-pass filter $\Phi(j \Omega)$ of $M$-channel are used to uniformly convert the input signals $\Phi(j \Omega)$ into $M$-channel baseband sub-signals of equal bandwidth, and thus the bandwidths of various sub-signals are reduced to $(1 / 2 M)$ of the input signals; then, the base band signal can be digitized by Mchannel ADC with sampling period of $(1 / 2 \mathrm{M})$, and then the carrier frequencies of various baseband sub-signals are, respectively, recovered to the tuning frequency points of analogue quadrature demodulator at the front end of corresponding channels using digital up-conversion (DUC) [14] and added together to obtain the full digital domain information of input analogue signals. The digital up-conversion (DUC) consists of three operations, namely, $2 M$-fold interpolation, digital low-pass filtering with a frequency response of $\Psi\left(e^{j \omega}\right)$, and digital quadrature modulation.

Full-domain processing is made for the signals in the first Nyquist domain, and then the tuning frequency of each channel analogue quadrature demodulator can be expressed as

$$
\vec{\Omega}=\left\{\Omega_{0}, \ldots, \Omega_{m}, \ldots, \Omega_{M-1}\right\}, \quad m \in[0, M-1],
$$

where $\Omega_{m}=(2 m+1) \cdot(\pi / 2 M)$.

The time-domain expression of analogue quadrature demodulator is

$$
O C_{m}=e^{-j \Omega_{m} t}=\cos \left(\Omega_{m} t\right)-j \sin \left(\Omega_{m} t\right) .
$$

According to Fourier transform characteristics, the signals $X_{m}(j \Omega)$ after analogue quadrature demodulation are as follows:

$$
X_{m}(j \Omega)=X\left(j \Omega+j \Omega_{m}\right)
$$

After sampling by analogue low-pass filter and ADC, the frequency characteristics of signals can be expressed as follows:

$$
\begin{aligned}
X_{m}\left(e^{j \omega}\right)= & \frac{1}{2 M T_{s}} \sum_{r=0}^{2 M-1} \\
& {\left[X\left(j \frac{\omega}{2 M T_{s}}+j \Omega_{m}-j \frac{\pi r}{M T_{s}}\right) \times \Phi\left(j \frac{\omega}{2 M T_{s}}-j \frac{\pi r}{M T_{s}}\right)\right] . }
\end{aligned}
$$

If $\omega_{m}$ is used to express the tuning frequency of subsequent digital quadrature modulator, then $\omega_{m}=\Omega_{m} T_{s}$ and the output signal $Y\left(e^{j \omega}\right)$ is

$$
Y\left(e^{j \omega}\right)=\sum_{r=0}^{2 M-1} X\left(j \frac{\omega}{T_{s}}-j \frac{\pi r}{M T_{s}}\right) \cdot T_{r}\left(e^{j \omega}\right),
$$

where

$$
T_{r}\left(e^{j \omega}\right)=\frac{1}{2 M T_{s}} \sum_{m=0}^{M-1} \Phi\left[j \frac{\omega}{T_{s}}-j \Omega_{m}-j \frac{\pi r}{M T_{s}}\right] \Psi\left(e^{j\left(\omega-\omega_{m}\right)}\right) .
$$

It expresses the transfer function of the system [15]. Assuming the output signal $Y\left(e^{j \omega}\right)$ is reproduction of undistorted digital domain of the input signal $X(j \Omega)$, then $T_{r}\left(e^{j \omega}\right)$ shall satisfy

$$
T_{r}\left(e^{j \omega}\right)= \begin{cases}C e^{-j \omega d}, & r=0, \\ 0, & r \in[1,2 M-1] .\end{cases}
$$

At this point, the output signal $Y\left(e^{j \omega}\right)=C e^{-j \omega d}$ $X\left(j\left(\omega / T_{s}\right)\right)$ is only the result of grain and delay effects of the input signal and there are no other spurious or aliasing phenomena. However, in fact, when $r \neq 0, T_{r}\left(e^{j \omega}\right)$ is impossible to be theoretically zero. This means that the reconstruction error exists objectively, which can only be reduced as far as possible to meet the signal-to-noise distortion ratio required by the intended design.

\section{Analysis of Analogue IQ Imbalance Errors}

This paper focuses on IQ imbalance analysis of analogue demodulation, so the effects of analogue device error, channel mismatch error, and signal reconstruction error are ignored for the time being.

Suppose the IQ imbalance errors are uncorrelated with the input signal's frequency; then, formula (2) is changed while the IQ imbalance errors are introduced: 


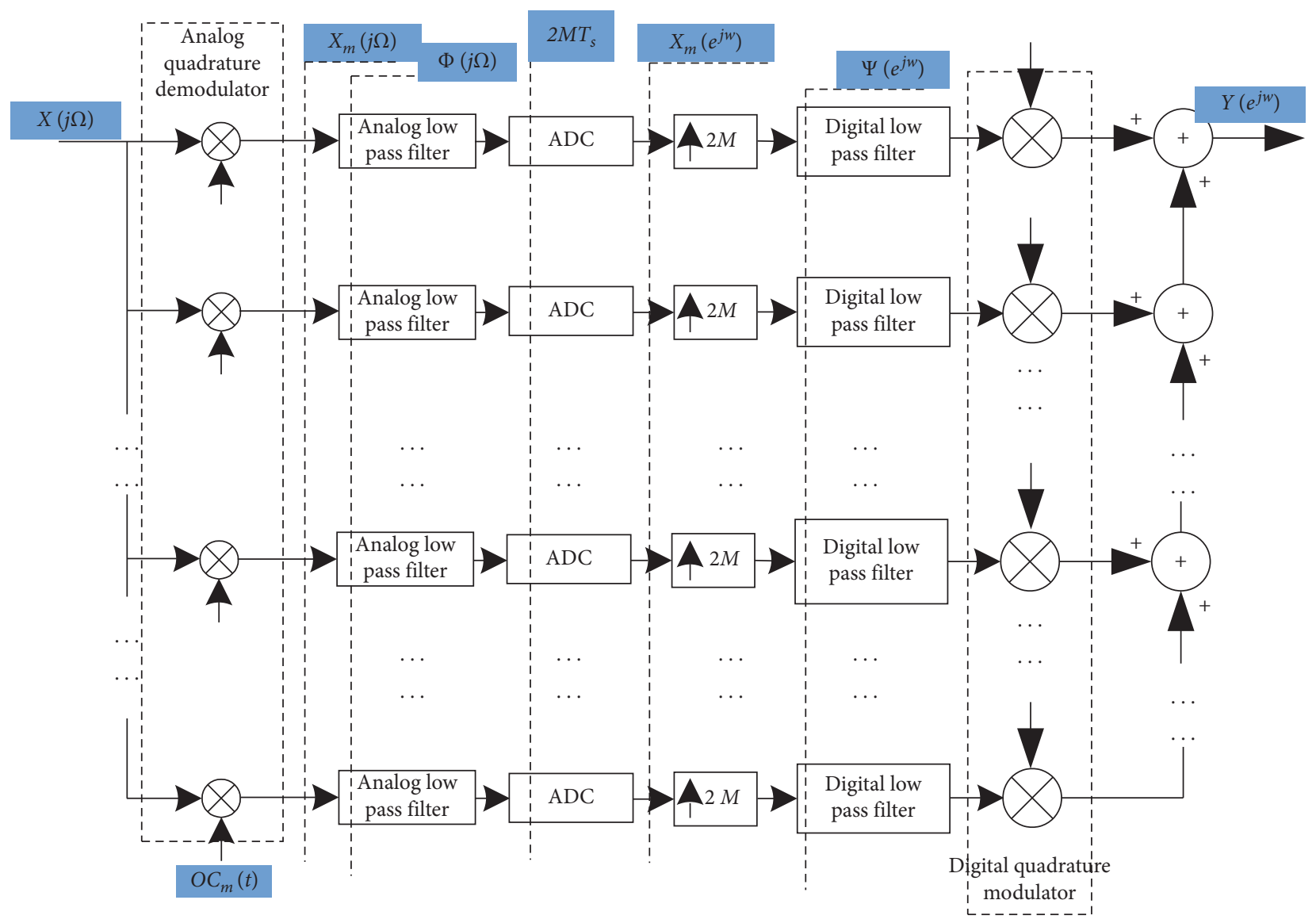

FIgURE 1: Principle of orthogonal frequency alternating acquisition systems.

$$
O C_{m}^{\Delta}=\cos \left(\Omega_{m} t\right)-j\left(1+\alpha_{m}\right) \sin \left(\Omega_{m} t+\varphi_{m}\right),
$$

where $\alpha_{m}$ and $\varphi_{m}$, respectively, express the magnitude imbalance parameter and phase imbalance parameter between the two channels $\mathrm{I}$ and $\mathrm{Q}$ in the $m^{\text {th }}$ channel analogue quadrature demodulator.

The Fourier transform expression of formula (8) is

$$
\begin{aligned}
F\left[\mathrm{OC}_{m}^{\Delta}\right]= & 2 \pi \delta\left(\Omega-\Omega_{m}\right)+\pi\left[1-\left(1+\alpha_{m}\right) \cos \varphi_{m}\right. \\
& \left.-j\left(1+\alpha_{m}\right) \sin \varphi_{m}\right] \delta\left(\Omega-\Omega_{m}\right) \\
& -\pi\left[1-\left(1+\alpha_{m}\right) \cos \varphi_{m}+j\left(1+\alpha_{m}\right) \sin \varphi_{m}\right] \delta \\
& \cdot\left(\Omega+\Omega_{m}\right),
\end{aligned}
$$

where $F[\cdot]$ represents Fourier operator. Then, in this case, the signal after analogue quadrature demodulation is updated as $X_{m}^{\Delta}(j \Omega)$, and

$$
\begin{aligned}
X_{m}^{\Delta}(j \Omega)= & X\left(j \Omega+j \Omega_{m}\right)+\frac{1-P_{m}}{2} X\left(j \Omega-j \Omega_{m}\right) \\
& +\frac{S_{m}-1}{2} X\left(j \Omega+j \Omega_{m}\right),
\end{aligned}
$$

where $P_{m}=\left(1+\alpha_{m}\right) e^{j \varphi_{m}}$ and $S_{m}=\left(1+\alpha_{m}\right) e^{-j \varphi_{m}}$. The signal after sampling by analogue low-pass filter and ADC is updated as $X_{m}^{\Delta}\left(e^{j \omega}\right)$ and

$$
\begin{aligned}
X_{m}^{\Delta}\left(e^{j \omega}\right)= & \frac{1}{2 M T_{s}} \sum_{r=0}^{2 M-1}\left[X\left(j \frac{\omega}{2 M T_{s}}+j \Omega_{m}-j \frac{\pi r}{M T_{s}}\right)+\frac{1-P_{m}}{2} X\left(j \frac{\omega}{2 M T_{s}}-j \Omega_{m}-j \frac{\pi r}{M T_{s}}\right)\right. \\
& \left.+\frac{S_{m}-1}{2} X\left(j \frac{\omega}{2 M T_{s}}+j \Omega_{m}-j \frac{\pi r}{M T_{s}}\right)\right] \times \Phi\left(j \frac{\omega}{2 M T_{s}}-j \frac{\pi r}{M T_{s}}\right) .
\end{aligned}
$$


Then, the output signal is updated as $Y^{\Delta}\left(e^{j \omega}\right)$ and satisfies

$$
Y^{\Delta}\left(e^{j \omega}\right)=Y\left(e^{j \omega}\right)+\partial_{m}
$$

where $\partial_{m}$ represents the error term of the system output signal with IQ imbalance compared with the output signal without IQ imbalance, and it meets

$$
\begin{aligned}
\partial_{m}= & \sum_{r=0}^{2 M-1}\left[X\left(j \frac{\omega}{T_{s}}-j \frac{\pi r}{M T_{s}}\right) \times \frac{1}{2 M T_{s}} \sum_{m=0}^{M-1} \frac{S_{m}-1}{2} \Phi\left(j \frac{\omega}{T_{s}}-j \Omega_{m}-j \frac{\pi r}{M T_{s}}\right) \Psi\left(e^{j \omega-j \omega_{m}}\right)\right] \\
& +\frac{1}{2 M T_{s}} \sum_{r=0}^{2 M-1} \sum_{m=0}^{M-1}\left[\frac{1-P_{m}}{2} X\left(j \frac{\omega}{T_{s}}-j 2 \Omega_{m}-j \frac{\pi r}{M T_{s}}\right) \times \Phi\left(j \frac{\omega}{T_{s}}-j \Omega_{m}-j \frac{\pi r}{M T_{s}}\right) \Psi\left(e^{j \omega-j \omega_{m}}\right)\right] .
\end{aligned}
$$

Obviously, $\partial_{m}$ directly reflects the spurious situation in the case of IQ imbalance.

In order to further evaluate the specific impact of am, the input signal is loaded as a single tone sinusoidal signal.
Suppose the amplitude is $U_{I N}$, and $\Omega_{I N}$ and $\omega_{I N}$ are the analogue and angular frequencies, respectively. At this point, formula (13) can be simplified into

$$
\begin{aligned}
\partial_{m}= & \sum_{r=0}^{2 M-1}\left\{\pi U_{I N}\left[\delta\left(\omega+\omega_{I N}-\frac{\pi r}{M}\right)+\delta\left(\omega-\omega_{I N}-\frac{\pi r}{M}\right)\right] \times \sum_{m=0}^{M-1} \frac{S_{m}-1}{2} \Phi\left(j \frac{\omega}{T_{s}}-j \Omega_{m}-j \frac{\pi r}{M T_{s}}\right) \Psi\left(e^{j \omega-j \omega_{m}}\right)\right\} \\
& +\sum_{r=0}^{2 M-1} \sum_{m=0}^{M-1}\left\{\frac{1-P_{m}}{2} \pi U_{I N}\left[\delta\left(\omega+\omega_{I N}-2 \omega_{m}-\frac{\pi r}{M}\right)+\delta\left(\omega-\omega_{I N}-2 \omega_{m}-\frac{\pi r}{M}\right)\right]\right. \\
& \left.\times \Phi\left(j \frac{\omega}{T_{s}}-j \Omega_{m}-j \frac{\pi r}{M T_{s}}\right) \times \Psi\left(e^{j \omega-j \omega_{m}}\right)\right\} .
\end{aligned}
$$

In formula (14), according to the frequency response characteristics, the analogue low-pass filter and the digital low-pass filter should have

$$
\left.\Phi\left(j \frac{\omega}{T_{s}}-j \Omega_{m}-j \frac{\pi r}{M T_{s}}\right) \Psi\left(e^{j \omega-j \omega_{m}}\right)\right|_{\omega= \pm \omega_{I N}+(\pi r / M)}=\Phi\left( \pm j \frac{\omega_{I N}}{T_{s}}-j \Omega_{m}\right) \Psi\left(e^{ \pm j \omega_{I N}-j \omega_{m}+(j \pi r / M)}\right) \begin{cases}\neq 0, & r=1 \\ \approx 0, & r>1\end{cases}
$$

Therefore, analysis for $\partial_{m}$ mainly focuses on the cases of $r=0$ and $r=1$. At this point, 


$$
\begin{aligned}
\left.\partial_{m}\right|_{r=0}= & \pi U_{I N}\left[\delta\left(\omega+\omega_{I N}\right)+\delta\left(\omega-\omega_{I N}\right)\right] \times \sum_{m=0}^{M-1} \frac{S_{m}-1}{2} \Phi\left(j \frac{\omega}{T_{s}}-j \Omega_{m}\right) \Psi\left(e^{j \omega-j \omega_{m}}\right) \\
& +\sum_{m=0}^{M-1}\left\{\frac{1-P_{m}}{2} \pi U_{I N}\left[\delta\left(\omega+\omega_{I N}-2 \omega_{m}\right)+\delta\left(\omega-\omega_{I N}-2 \omega_{m}\right)\right] \times \Phi\left(j \frac{\omega}{T_{s}}-j \Omega_{m}\right) \times \Psi\left(e^{j \omega-j \omega_{m}}\right)\right\}, \\
\left.\partial_{m}\right|_{r=1}= & \pi U_{I N}\left[\delta\left(\omega+\omega_{I N}-\frac{\pi}{M}\right)+\delta\left(\omega-\omega_{I N}-\frac{\pi}{M}\right)\right] \times \sum_{m=0}^{M-1} \frac{S_{m}-1}{2} \Phi\left(j \frac{\omega}{T_{s}}-j \Omega_{m}-j \frac{\pi}{M T_{s}}\right) \Psi\left(e^{j \omega-j \omega_{m}}\right) \\
& +\sum_{m=0}^{M-1}\left\{\frac{1-P_{m}}{2} \pi U_{I N}\left[\delta\left(\omega+\omega_{I N}-2 \omega_{m}-\frac{\pi}{M}\right)+\delta\left(\omega-\omega_{I N}-2 \omega_{m}-\frac{\pi}{M}\right)\right] \times \Phi\left(j \frac{\omega}{T_{s}}-j \Omega_{m}-j \frac{\pi}{M T_{s}}\right) \times \Psi\left(e^{j \omega-j \omega_{m}}\right)\right\} .
\end{aligned}
$$

According to formulas (16) and (17), it can be founded that $\partial_{m}$ will produce excessive frequency spectrum signals at the frequency position ${ }_{\omega}^{I Q}$, and

$\omega_{I Q}=\left\{ \pm \omega_{I N}+\frac{\pi}{M}\right\} \cup\left\{ \pm \omega_{I N}+2 \omega_{m}\right\} \cup\left\{ \pm \omega_{I N}+\frac{\pi}{M}+2 \omega_{m}\right\}$.

Formula (18) directly reflects the frequency distribution of spurious signals brought about by imbalance error, and the position where it appears is closely related to the input signal frequency and the tuning frequency of quadrature modulation and demodulation, and the number of spurious signals is obviously more than that of spurious signals caused by grain error, biased error, and time error of ADC acquisition channel. The reason mainly lies in that the IQ mismatch errors bring an additional impact factor (i.e., the tuning frequency of quadrature modulation) to the channel mismatch.

To further analyze the amplitude strength of spurious signals, now a uniform n-point discrete Fourier transform is made for formula (4) within the interval of $[0,2 \pi]$. At this point, $r=0$; therefore,

$$
\begin{aligned}
\left.X_{m}^{\Delta}\left(e^{j \omega}\right)\right|_{\omega=(2 \pi k / N)}= & \pi U_{I N}\left\{\begin{array}{r}
{\left[\left(1+\frac{S_{m}-1}{2}\right) \delta\left(\frac{2 \pi k}{N}+2 M \omega_{I N}+2 M \omega_{m}\right)+\left(1+\frac{S_{m}-1}{2}\right) \delta\left(\frac{2 \pi k}{N}-2 M \omega_{I N}+2 M \omega_{m}\right)\right]} \\
+\frac{1-P_{m}}{2}\left[\left(\frac{2 \pi k}{N}+2 M \omega_{I N}-2 M \omega_{m}\right)+\delta\left(\frac{2 \pi k}{N}-2 M \omega_{I N}-2 M \omega_{m}\right)\right]
\end{array}\right\} \\
& \times \Phi\left(j \frac{\pi k}{M N T_{s}}\right) .
\end{aligned}
$$

In formula (19), $k \in[0, N-1] . X_{m}^{\Delta}\left(e^{j \omega}\right)$ represents the frequency spectrum characteristics of signals after sampling by ADC, and the frequency spectrum value of each point is measurable and then the amplitude imbalance parameter $\alpha_{m}$ and phase imbalance parameter $\varphi_{m}$ between the two analogue channels I and Q can be estimated in the inverse direction according to the number of frequency spectrum of signals after sampling by ADC. Details are as follows.

When $k=\left(\omega_{I N}-\omega_{m} / \pi\right) \cdot N \cdot M$, it can be obtained that

$$
\begin{aligned}
\left.X_{m}^{\Delta}\left(e^{j \omega}\right)\right|_{\omega=(2 \pi k / N)}= & \pi U_{I N}\left(1+\frac{S_{m}-1}{2}\right) \Phi \\
& \left(j \Omega_{I N}-j \Omega_{m}\right) .
\end{aligned}
$$

When $k=\left(\omega_{I N}-\omega_{m} / \pi\right) \cdot N \cdot M$, it can be obtained that

$$
\left.X_{m}^{\Delta}\left(e^{j \omega}\right)\right|_{\omega=(2 \pi k / N)}=\pi U_{I N}\left(1+\frac{1-P_{m}}{2}\right) \Phi\left(j \Omega_{I N}+j \Omega_{m}\right) .
$$

Then, combining formulas (20) and (21), it can be obtained that

$$
\left\{\begin{array}{l}
\left(1+\alpha_{m}\right) e^{-j \varphi_{m}}=\frac{2 X_{m}^{\Delta}\left(e^{j \omega}\right)}{\pi U_{I N} \Phi\left(j \Omega_{I N}-j \Omega_{m}\right)}-1, \\
\left(1+\alpha_{m}\right) e^{j \varphi_{m}}=3-\frac{2 X_{m}^{\Delta}\left(e^{j \omega}\right)}{\pi U_{I N} \Phi\left(j \Omega_{I N}+j \Omega_{m}\right)} .
\end{array}\right.
$$

In formula (22), $\Phi\left(j \Omega_{I N}+j \Omega_{m}\right)$ can be obtained according to the designed analogue low-pass filter $\Phi(j \Omega)$. According to formula (22), the computation expressions of amplitude imbalance and phase imbalance parameters can be obtained as follows: 


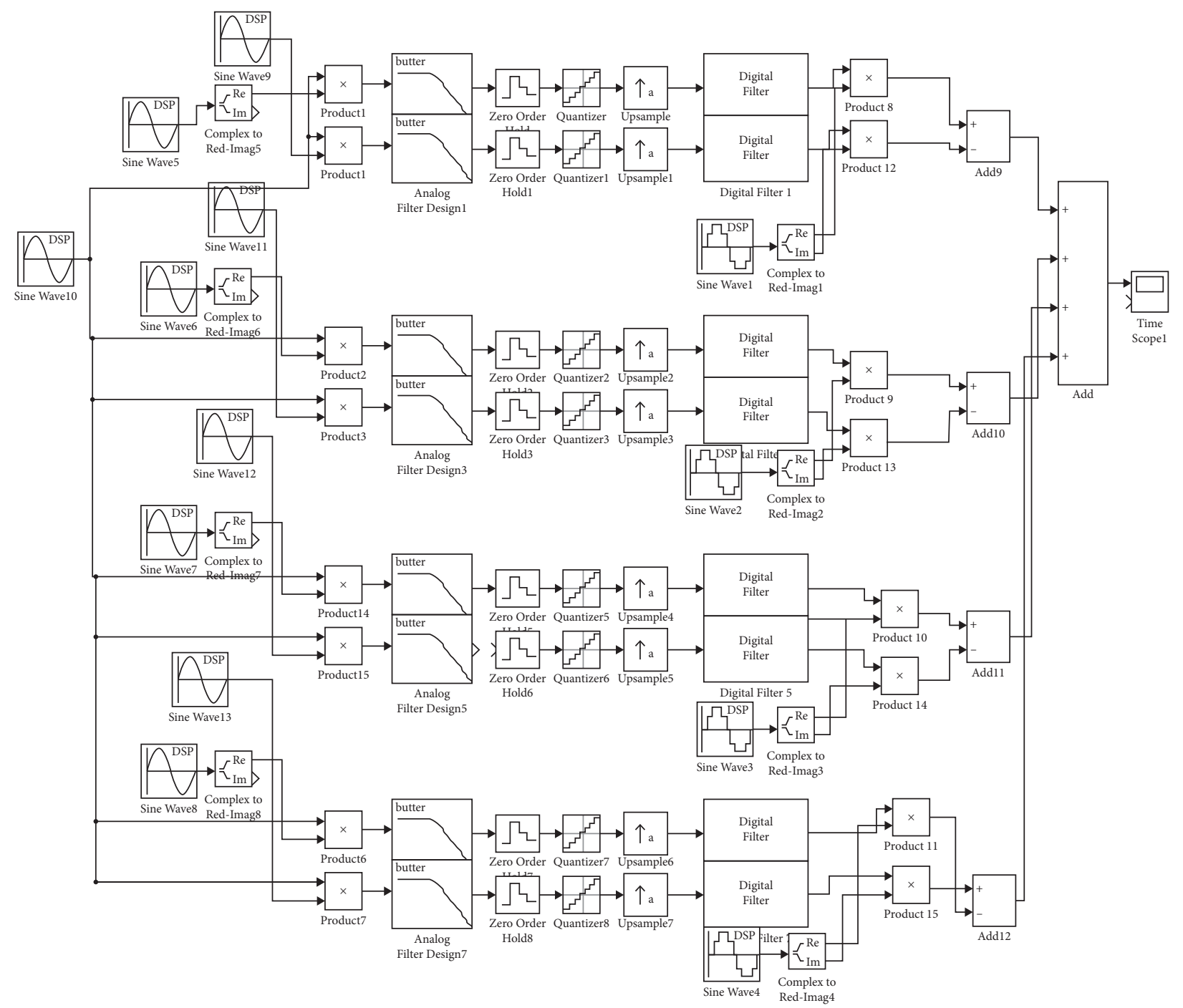

FIGURE 2: Simulink model of 4-channel high-speed system with IQ mismatch.

$$
\left\{\begin{array}{l}
\alpha_{m}=\sqrt{\Pi \times \amalg}-1, \varphi_{m}=\frac{\Theta}{2 \Gamma}, \\
\Pi=\frac{2\left|X_{m}^{\Delta}\left(e^{j \omega}\right)\right|}{\pi U_{I N}\left|\Phi\left(j \Omega_{I N}-j \Omega_{m}\right)\right|}-1, \\
\amalg=3-\frac{2\left|X_{m}^{\Delta}\left(e^{j \omega}\right)\right|}{\pi U_{I N}\left|\Phi\left(j \Omega_{I N}-j \Omega_{m}\right)\right|}, \\
\Theta=\theta\left[3-\frac{2 X_{m}^{\Delta}\left(e^{j \omega}\right)}{\pi U_{I N} \Phi\left(j \Omega_{I N}-j \Omega_{m}\right)}\right], \\
\Gamma=\theta\left[\frac{2 X_{m}^{\Delta}\left(e^{j \omega}\right)}{\pi U_{I N} \Phi\left(j \Omega_{I N}-j \Omega_{m}\right)}-1\right] .
\end{array}\right.
$$




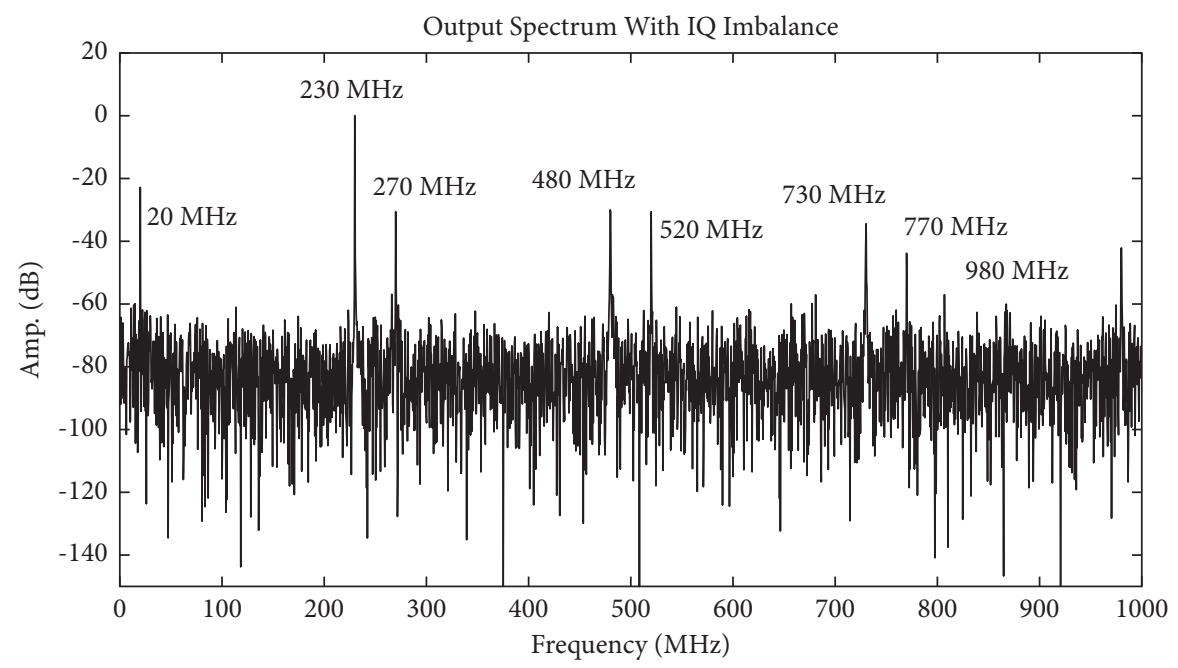

FIgURE 3: Output spectrum under the effect of IQ mismatch.

TABLE 1: Statistics of spurious source.

\begin{tabular}{lr}
\hline Spurious frequency point $(\mathrm{MHz})$ & Spurious source \\
\hline 20 & $-f_{I N}+\left(f_{s} / 2 M\right),-f_{I N}+2 f_{0},-f_{I N}+2 f_{2}$ \\
270 & $-f_{I N}+\left(f_{s} / 2 M\right)+2 f_{0},-f_{I N}+\left(f_{s} / 2 M\right)+2 f_{2}$ \\
480 & $f_{I N}+\left(f_{s} / 2 M\right), f_{I N}+2 f_{0}, f_{I N}+2 f_{2}$ \\
520 & $-f_{I N}+2 f_{1},-f_{I N}+2 f_{3}$ \\
730 & $f_{I N}+\left(f_{s} / 2 M\right)+2 f_{0}, f_{I N}+\left(f_{s} / 2 M\right)+2 f_{2}$ \\
770 & $-f_{I N}+\left(f_{s} / 2 M\right)+2 f_{1},-f_{I N}+\left(f_{s} / 2 M\right)+2 f_{3}$ \\
980 & $f_{I N}+2 f_{1}, f_{I N}+2 f_{3}$ \\
\hline
\end{tabular}

\section{Simulation Verification}

To verify that the IQ imbalance error in the complete orthogonal frequency alternating acquisition system can bring about spurious effects on the output signal, experimental analysis is further made using the Simulink simulation tool. The Simulink model is shown in Figure 2. Here, the complete orthogonal frequency alternating acquisition system is instantiated as a four-channel $(M=4)$ high-speed acquisition system, with a total sampling rate of $f_{s}=2 G S P S$ $\left(f_{s}=\left(1 / T_{S}\right)\right)$, which totally requires eight low-speed ADCs operating at $250 \mathrm{MHz}$.

Then, the quadrature modulation and demodulation frequencies $f_{m}\left(f_{m}=\omega_{m} / 2 \pi=\left(\Omega_{m} T_{s} / 2 \pi\right)\right)$ of various channels are $f_{0}=125 \mathrm{MHz}, f_{1}=375 \mathrm{MHz}, f_{2}=625 \mathrm{MHz}$, and $f_{3}=875 \mathrm{MHz}$, respectively.

Let the input signal be a cosine signal, and it is expressed as follows:

$$
x(t)=\cos \left(2 \pi f_{I N} t\right)
$$

where $f_{I N}=\left(\omega_{I N} / 2 \pi\right)=\left(\Omega_{I N} T_{s} / 2 \pi\right)$. To significantly verify the spurious effects of amplitude imbalance and phase imbalance, the amplitude imbalance parameters of various channels are set as 8, 9, 10, and 11, respectively, and the phase imbalance parameters of various channels are set as $(\pi / 6),(\pi / 8),(\pi / 10)$, and $(\pi / 12)$, respectively. Meanwhile, set $f_{I N}=230 \mathrm{MHz}$. The frequency spectrum of the output signal is obtained as shown in Figure 3.
In the frequency spectrum information as shown in Figure 3, in addition to the input information including $230 \mathrm{MHz}$, there are also a total of seven spurious signals at $20 \mathrm{MHz}, 270 \mathrm{MHz}, 480 \mathrm{MHz}, 520 \mathrm{MHz}, 730 \mathrm{MHz}, 770 \mathrm{MHz}$, and $980 \mathrm{MHz}$.

Here, formula (18) is rewritten as the frequency form:

$$
f_{I Q}=\left\{ \pm f_{I N}+\frac{f_{s}}{2 M}\right\} \cup\left\{ \pm f_{I N}+2 f_{m}\right\} \cup\left\{ \pm f_{I N}+\frac{f_{s}}{2 M}+2 f_{m}\right\} \text {. }
$$

Calculation based on formula (25) and various setting parameters reveals that the spurious signals theoretically deduced are all reflected in these seven signals (see Table 1 for details).

Thus, it can be seen that the simulation verification results are consistent with the theoretical derivation, reflecting the objective existence of spurious effects caused by IQ imbalance.

\section{Conclusion}

The complete orthogonal frequency alternating acquisition systems are a kind of high-speed signal processing systems built based on low-speed narrowband analogue-to-digital converter devices, with the advantages of lower sampling rate of required devices, simpler filter design, and easier engineering application than the traditional frequency-interleaved acquisition systems. However, due to the different 
analog quadrature demodulation operation of this kind of system, there are not only the same analog implementation error and channel mismatch error as the traditional system but also IQ imbalance error. In order to master the effects of IQ imbalance errors on such systems, this paper makes a detailed study and mathematically deduces the spurious effects of imbalance errors and verifies them combined with simulation experiments. As a whole, the IQ imbalance errors will bring several spurious signals to the output signals and cause frequency spectrum deterioration, and the frequency distribution of these spurious signals is directly influenced by the input signal frequency and the analogue quadrature demodulation frequency.

\section{Data Availability}

The data used to support the findings of this study are available from the corresponding author upon request.

\section{Conflicts of Interest}

The authors declare that they have no conflicts of interest.

\section{Acknowledgments}

This study was supported by the Chengdu Science and Technology Bureau Project (2019-YF05-02118-SN).

\section{References}

[1] E. J. Candes, J. Romberg, and T. Tao, "Robust uncertainty principles: exact signal reconstruction from highly incomplete frequency information," IEEE Transactions on Information Theory, vol. 52, no. 2, pp. 489-509, 2006.

[2] P. Yang and S. Su, "High speed high precision ADCsystem based on time interleaving technology," Electron Device, vol. 48, no. 39, pp. 1397-1401, 2016.

[3] L. Qiu, Y. Zheng, and L. Siek, "Analysis and design of high performance frequency-interleaved ADC," in Proceedings of the IEEE International Symposium on Circuits \& Systems, pp. 2022-2025, Beijing, China, May 2013.

[4] Xu Liu and Y. Zhao, "A novel oversampling scheme for design of hybrid filter bank based ADCs," IEICE Electronics Express, vol. 15, Article ID 20180007, 2018.

[5] J. A. Tropp and A. C. Gilbert, "Signal recovery from random measurements via orthogonal matching pursuit," IEEE Transactions on Information Theory, vol. 53, no. 12, pp. 4655-4666, 2007.

[6] W. Wang and Z. Zhang, "Sampling technology of wide-band signals based on modulated hybrid filter banks," Signal Processing, vol. 30, no. 10, pp. 1185-1192, 2014.

[7] T. Liu, K. Lian, L. Ye, and S. Tian, "Research on an orthogonal frequency-interleaved sampling and reconstruction method," Journal of University of Electronic Science and Technology of China, vol. 48, no. 6, pp. 858-864, 2019.

[8] X. Peng, J. Li, X. Zhou, Q. Lin, and Z. Chen, "Analysis and design of M-channel hybrid filter bank with digital calibration," IEEE Access, vol. 6, Article ID 24606, 2018.

[9] E. J. Candès, J. K. Romberg, and T. Tao, "Stable signal recovery from incomplete and inaccurate measurements," Communications on Pure and Applied Mathematics, vol. 59, no. 8, pp. 1207-1223, 2006.
[10] S. L. Su, S. P. Hung, and J. Lin, "IQ imbalance compensation," Journal of the Korean Society for Aeronautical \& Space Sciences, vol. 48, no. 11, pp. 861-871, 2020.

[11] T. Liu, S. Tian, Z. Wang, and L. Guo, "Analysis and design of $M$-channel frequency-interleaved ADC with analog filter estimation," Analog Integrated Circuits and Signal Processing, vol. 81, no. 1, pp. 173-180, 2014.

[12] H. Li and Y. Qian, "Influence of IQ channel imbalance on simultaneous transmission and reception based cognitive antijamming system," Journal of Beijing University of Aeronautics and Astronautics, vol. 43, no. 3, pp. 449-456, 2017.

[13] X. Zhan, P. Liu, S. Ren, and Y. Cheng, "IQ imbalance evaluation and compensation method of ultra-wideband receiver," Chinese Journal of Scientific Instrument, vol. 39, no. 6, pp. 157-163, 2018.

[14] H. Ye, K. Su, L. Wu, and Y. Weng, "Digital up-converter circuit for all-digital transmitter," Journal of Fuzhou University (nature science version), vol. 44, no. 5, pp. 649-655, 2016.

[15] E. Nayebi, P. Dayal, and K. B. Song, "Adaptive IQ mismatch compensation in time-domain using frequency-domain observations," IEEE Transactions on Signal Processing, vol. 69, no. 99 , p. $1,2021$. 